\title{
Características de la epidemia de cólera de 1998 en Ecuador, durante el fenómeno de "El Niño"
}

\author{
Jean-Marc Gabastou, ${ }^{1}$ Carmen Pesantes, ${ }^{2}$ Santiago Escalante, ${ }^{3}$ Yolanda \\ Narváez, ${ }^{2}$ Enrique Vela, ${ }^{3}$ Lidia García, ${ }^{4}$ Diana Zabala ${ }^{5}$ y Zaida E. Yadon ${ }^{6}$
}

RESUMEN Objetivos. Caracterizar el brote de cólera ocurrido en Ecuador en 1998 durante el fenómeno de "El Niño", presentar los datos sobre la resistencia de las cepas circulantes de Vibrio cholerae a los antimicrobianos y describir las medidas preventivas tomadas por las autoridades sanitarias para reducir el impacto de la enfermedad.

Métodos. Los datos epidemiológicos provienen de los registros de la Dirección Nacional de Epidemiología del Ministerio de Salud Pública de Ecuador y del Instituto Nacional de Higiene y Medicina Tropical, y el informe final del Programa de Formación para la Lucha contra el Cólera y las Enfermedades Diarreicas (PROCED ALA 93/25). Se procedió a aislar, identificar y serotipificar $\mathrm{V}$. cholerae en las muestras de heces de 10\% de los pacientes con posible cólera identificados entre el 1 de enero y el 31 de diciembre de 1998. Los casos sospechados se definieron por la aparición súbita de diarrea acuosa, con o sin deshidratación, en zonas epidémicas. Las cepas aisladas se sometieron a un antibiograma estándar por el método de difusión, en el que se probaron los siguientes antibióticos: amoxicilina, tetraciclina, sulfametoxazol con trimetoprim, compuesto vibriostático O/129, ácido nalidíxico, eritromicina, norfloxacino, ciprofloxacino, gentamicina, cloranfenicol y colistina.

Resultados. En 1998 se notificaron 3755 casos en 17 de las 21 provincias del país, lo que corresponde a una tasa de incidencia de 53,96 por 100000 habitantes. Treinta y siete pacientes fallecieron, lo cual supone una tasa de letalidad del 0,97\%. Se aislaron 301 cepas de $\mathrm{V}$. cholerae en las 637 muestras con sospechosa de cólera que se procesaron; todas correspondieron a V. cholerae O:1, El Tor, subtipo Ogawa. La totalidad de las cepas fueron sensibles a la tetraciclina y a las quinolonas, y 5,6\% resistentes a la eritromicina. La única cepa resistente a la amoxicilina fue multirresistente. Las autoridades nacionales pusieron en práctica una serie de medidas preventivas en la comunidad y se fortaleció el sistema de vigilancia para reducir el impacto de la enfermedad.

Conclusiones. Las medidas preventivas contribuyeron a reducir el impacto de la nueva epidemia de cólera en el Ecuador, tanto en términos de letalidad como de incidencia. En vista de la sensibilidad global de las cepas a los antimicrobianos, no se justifica cambiar los esquemas de tratamiento vigentes en la Región. Teniendo en cuenta la frecuencia de los desastres naturales en este país y su relación con la reaparición del cólera, es recomendable diseñar intervenciones que permitan prevenir y controlar la reaparición de la enfermedad y su extensión hacia las provincias más vulnerables de la Sierra y el Oriente.

Palabras clave Cólera, prevención, resistencia a los antimicrobianos, vigilancia epidemiológica.

1 Programa de Formación para la Lucha contra el Cólera y las Enfermedades Diarreicas y Organización Panamericana de la Salud/Organización Mundial de la Salud (PROCED ALA/93/25 y OPS/OMS), Quito, Ecuador. Toda la correspondencia debe ser enviada a Jean-Marc Gabastou a la siguiente dirección: PAHO/WHO (HSP/HSE),
525, 23rd St. NW, Washington, D.C. 20037. Estados Unidos de América. Correo electrónico: gabastoj @paho.org

2 PROCED-Instituto Nacional de Higiene y Medicina Tropical (INHMT). Laboratorio de Bacteriología-Peste, INHMT, Guayaquil, Ecuador.

3 PROCED-INHMT, Quito, Ecuador.
4 Dirección Nacional de Epidemiología, Ministerio de Salud Pública, Quito, Ecuador.

5 PROCED, Quito, Ecuador.

6 OPS/OMS (HCP/HCT), Washington, D.C., Estados Unidos de América. 
Desaparecido desde 1895 en Latinoamérica, el cólera hizo su reaparición en la costa de Perú el 23 de enero de 1991 (1-3). El Vibrio cholerae O:1, El Tor, característico de la séptima pandemia mundial (4), se asoció a la difusión rápida de la enfermedad de la costa peruana a los países andinos vecinos y, luego, a la casi totalidad de América Latina, afectando a 21 países (5-8). En 1991, la epidemia culminó con 396533 casos en la Región de las Américas, de los cuales 4093 fallecieron (8). Perú y Ecuador fueron los países más afectados, con 322562 y 46284 casos, respectivamente (8). Después la epidemia disminuyó progresivamente hasta 1997, pero la enfermedad adquirió carácter endémico (9).

El cólera reapareció en la zona andina en 1998, con el fenómeno climático de "El Niño". En América Latina, el número de casos pasó de 17760 en 1997 a 57106 en 1998, de los cuales 558 fallecieron (10). Este nuevo brote afectó más específicamente a Perú y Ecuador, países donde el cólera queda como un problema preocupante de salud pública, sobre todo en zonas geográficas con difícil acceso a los servicios de salud (11).

Este artículo presenta las características del brote de cólera ocurrido en Ecuador en 1998, los datos sobre la resistencia de $V$. cholerae a los antimicrobianos y las medidas de prevención y control tomadas por las autoridades sanitarias.

\section{MATERIALES Y MÉTODOS}

\section{Recolección de datos epidemiológicos}

Los datos de este estudio provienen de tres fuentes: los registros de la Dirección Nacional de Epidemiología del Ministerio de Salud Pública (MSP) de Ecuador y del Instituto Nacional de Higiene y Medicina Tropical $(8,12$, 13), y el informe final del Programa de Formación para la Lucha contra el Cólera y las Enfermedades Diarreicas (PROCED ALA 93/25) 7 . Los casos con

\footnotetext{
7 Gabastou JM, Zabala D. PROCED ALA 93/25, informe final. 1999. Circulación restringida.
}

sospecha de cólera fueron notificados a las 21 direcciones provinciales de salud del país, las cuales, a su vez, notificaron a la Dirección Nacional de Epidemiología en Quito. La definición de caso sospechado se basó en la aparición súbita de diarrea acuosa, con o sin deshidratación, en zonas epidémicas. Los primeros 50 casos fueron confirmados por pruebas de laboratorio y luego, durante la epidemia, se recomendó enviar al laboratorio las muestras del $10 \%$ de los casos, para confirmación bacteriológica.

\section{Cepas bacterianas: aislamiento y caracterización}

Las muestras de heces de pacientes con sospecha de cólera obtenidas entre el 1 de enero y el 31 de diciembre de 1998 se sembraron en agar TCBS (tiosulfato, citrato, sales biliares y sacarosa) y agar alcalino para el aislamiento de Vibrio spp., y en agar de MacConkey, caldo de Mueller-Kauffmann y agar de Hektoen para el aislamiento de otras bacterias entéricas (14), y se incubaron a $37^{\circ} \mathrm{C}$ durante 18 horas. Estos procedimientos se realizaron en los laboratorios provinciales de salud pública de la red del Instituto Nacional de Higiene y Medicina Tropical (INHMT). Las colonias características, positivas para la oxidasa, se sembraron en tubos de Cary-Blair y se enviaron al laboratorio nacional de referencia, ubicado en las instalaciones del PROCED ALA 93/25 en Quito y Guayaquil, para confirmación diagnóstica. Para las pruebas bioquímicas, se utilizó el kit API-20E ${ }^{\circledR}$ (bioMérieux, Marcy l'Étoile, Francia). Las cepas identificadas como $V$. cholerae, El Tor, fueron sometidas a pruebas de serotipificación con antisuero polivalente O:1 y antisueros monovalentes Inaba y Ogawa (Difco, Detroit, EE. UU.). Los discos de compuesto vibriostático $\mathrm{O} / 129$ y colistina se usaron como confirmación diagnóstica en el antibiograma. Después de procesarlas, las cepas fueron congeladas a $-70{ }^{\circ} \mathrm{C}$ para estudios ulteriores de marcadores epidemiológicos por biología molecular, para compararlas con las cepas de las epidemias anteriores cuando se disponga de medios económicos para ello.

\section{Antibiogramas}

Todas las cepas de $V$. cholerae aisladas fueron objeto de un antibiograma estandarizado con el método de difusión en gelosa, utilizando el medio de Mueller-Hinton, $\mathrm{pH}$ 7,4, y discos de Sanofi Pasteur (Paris, Francia) (15) con amoxicilina, $25 \mu \mathrm{g}$; tetraciclina, 30 UI; trimetoprim, 1,25 $\mu \mathrm{g} /$ sulfametoxazol 23,75 $\mu \mathrm{g}$; compuesto vibriostático $\mathrm{O} / 129$; ácido nalidíxico, $30 \mu \mathrm{g}$; eritromicina, $15 \mathrm{UI}$; norfloxacino, $5 \mu \mathrm{g}$; ciprofloxacino, $5 \mu \mathrm{g}$; gentamicina, $15 \mu \mathrm{g}$; cloranfenicol, $30 \mu \mathrm{g}$, y colistina, $50 \mu \mathrm{g}$. Para definir las cepas sensibles, intermedias y resistentes a cada uno de los antibióticos probados se utilizaron los diámetros críticos $(\mathrm{mm})$ recomendados por el Comité Francés del Antibiograma (15). Para evaluar el grado de concordancia con los resultados publicados entre 1994 y 1996, 10\% de las cepas fueron procesadas según el método de Kirby-Bauer, vigente en la red nacional hasta $1997(16,17)$. Se probaron los mismos antibióticos, aplicando los criterios de interpretación y los controles de calidad de la prueba de difusión por discos, incluido el uso de cepas de la American Type Culture Collection, establecidos por el National Committee for Clinical Laboratory Standards (NCCLS) (16).

\section{RESULTADOS}

\section{Datos epidemiológicos}

Después de la fase epidémica que sufrió Ecuador entre 1991 y 1992, se instaló una fase endemoepidémica con una recrudescencia esporádica de casos durante la temporada de lluvias en la Costa y una difusión secundaria a la Sierra (18). En 1997, el número de casos no superó los 65 (8), pero en 1998, el fenómeno climático de "El Niño" favoreció la reaparición de la enfermedad (10) y se notificaron 3755 casos de cólera. 

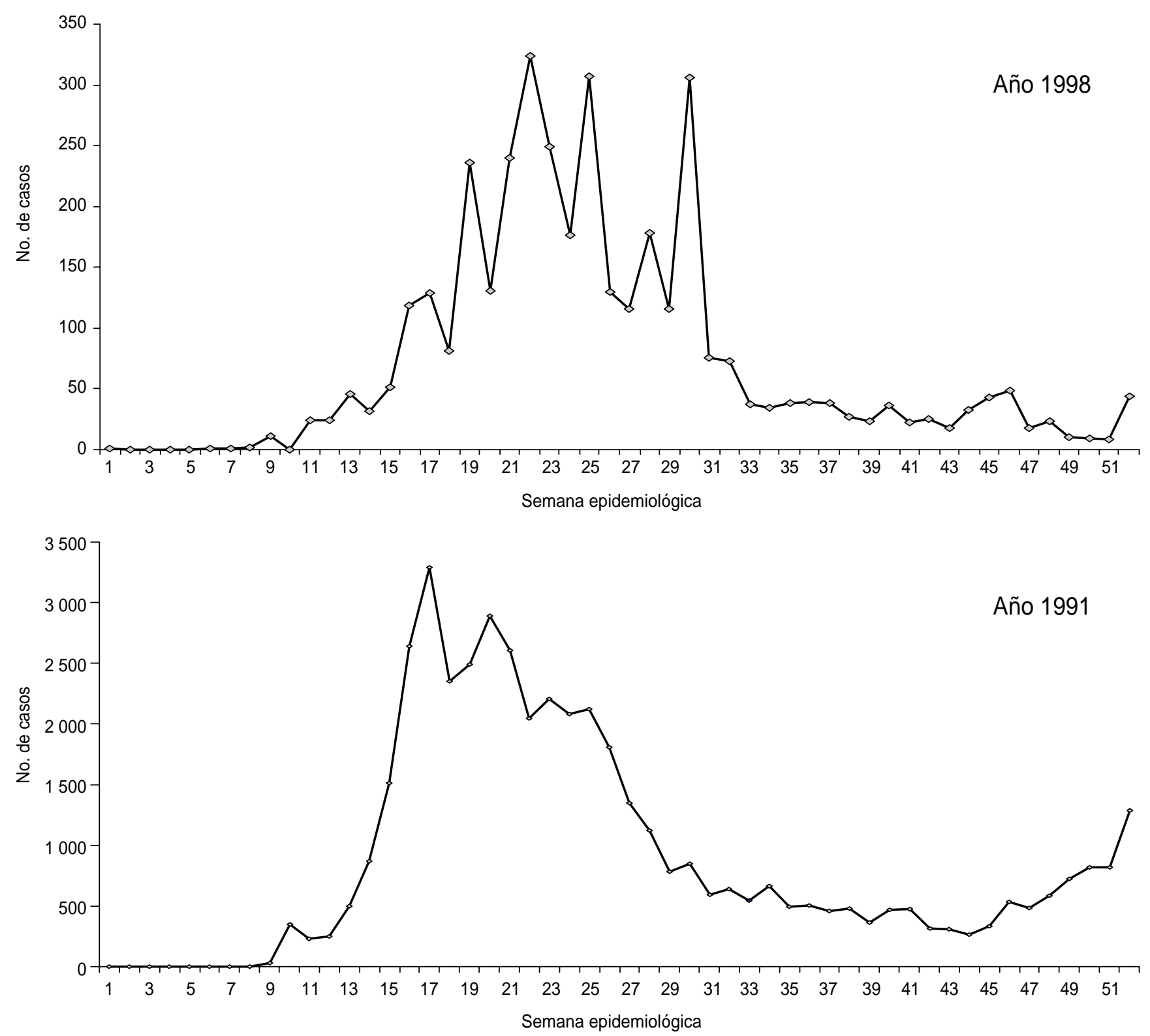

Fuente: Dirección Nacional de Epidemiología. Ministerio de Salud Pública de Ecuador.

La figura 1 muestra el número de casos de cólera por semana epidemiológica en las epidemias de 1998 y 1991. En 1998, el primer caso se detectó durante la primera semana de enero en la provincia de Loja, fronteriza con Perú (12). No se registraron más casos hasta el mes de febrero (sexta semana) en la misma provincia. Durante la novena semana, el cólera hizo su aparición en El Oro, provincia costera vecina de Loja (figura 2), a partir de la cual el brote se extendió de sur a norte a las otras provincias de la Costa y de oeste a este, afectando a la Sierra y al Oriente. La epidemia culminó en la semana 22 con 324 casos (12).

El cuadro 1 muestra el número de casos de cólera, la tasa anual de incidencia por 100000 habitantes y la tasa de letalidad, todo ello por provincias, durante las epidemias de $1998(12,13)$ y $1991(8,12)$. En 1998, los 3755 casos notificados correspondieron a una tasa de incidencia anual acumulada de 30,84 por 100000 habitantes. Treinta y siete pacientes fallecieron, lo cual supone una tasa de letalidad de $0,97 \%$. La enfermedad solo afectó a 17 de las 21 provincias del país. Se notificaron casos en todas las provincias de la Costa y la Sierra, pero solo en dos de las cinco provincias del Oriente; el archipiélago de las Galápagos, situado a mil kilómetros de la Costa, no se vio afectado. La Costa, con 3281 casos (tasa de inciden- 
FIGURA 2. Ecuador: provincias y regiones

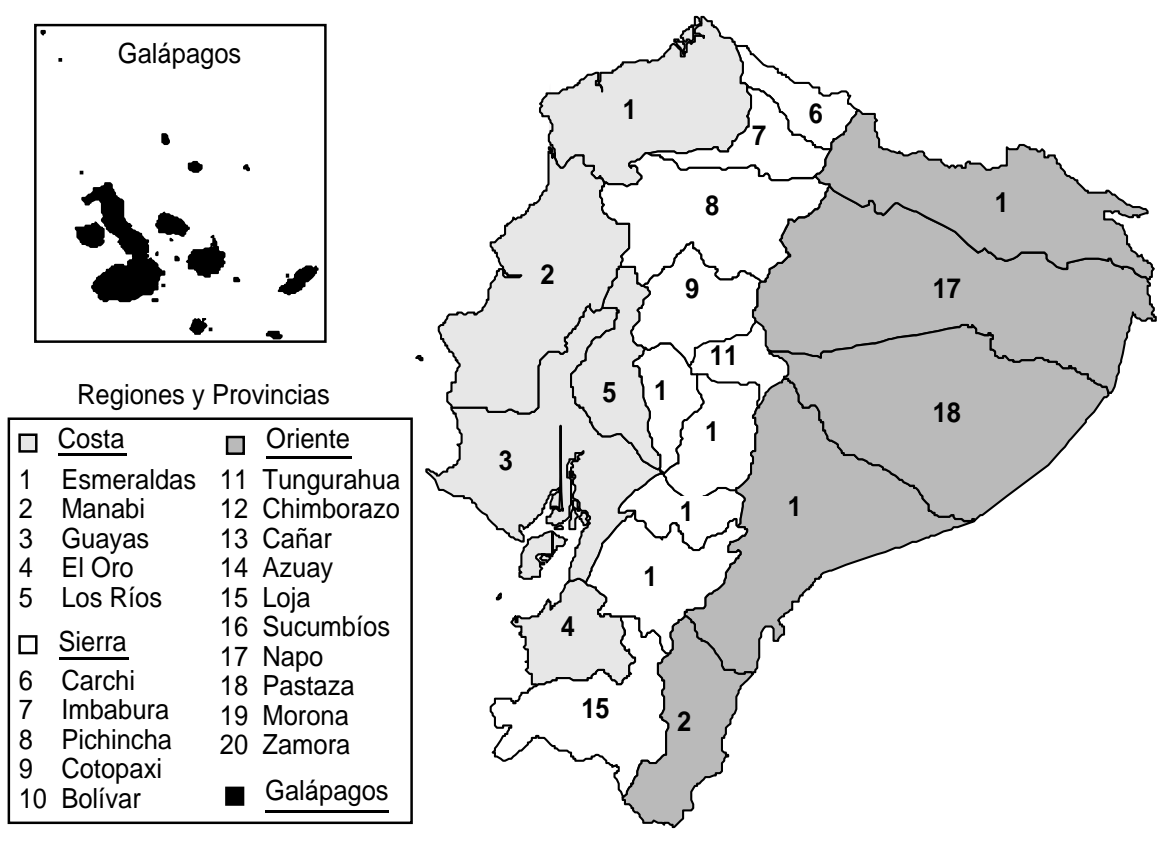

cia de 53,96 por 100000 ) habitantes, se vio más afectada que la Sierra, con 466 casos $(8,61$ por 100000$)$, y el Oriente, con 8 casos (1,39 por 100000$)$, confirmándose el tropismo de la enfermedad por la región costeña (8). Al contrario de la incidencia, la letalidad fue mayor en la Sierra (21 muertos; $4,5 \%$ ) que en la Costa (16 muertos; 0,5\%) (8). La letalidad mostró una gran disparidad entre las provincias, siendo Cañar y Carchi, ambas en la Sierra, las provincias con cifras más elevadas (cuadro 1) (12).

\section{Datos de laboratorio: caracterización de las cepas y sensibilidad a los antimicrobianos}

En la red de laboratorios del INHMT se procesaron 637 muestras con sospecha de cólera, aislándose 301 cepas de $V$. cholerae, 3 de $V$. parahaemolyticus, 2

CUADRO 1. Distribución de los casos notificados de cólera, su incidencia y letalidad, según provincia. Ecuador, 1991 y 1998

\begin{tabular}{|c|c|c|c|c|c|c|c|c|}
\hline \multirow[b]{3}{*}{ Provincia } & \multicolumn{4}{|c|}{1991} & \multicolumn{4}{|c|}{1998} \\
\hline & \multirow[b]{2}{*}{ No. de casos } & \multirow[b]{2}{*}{ Incidencia ${ }^{a}$} & \multicolumn{2}{|c|}{ Letalidad } & \multirow[b]{2}{*}{ No. de casos } & \multirow[b]{2}{*}{ Incidencia $^{a}$} & \multicolumn{2}{|c|}{ Letalidad } \\
\hline & & & No. & $(\%)$ & & & No. & $\%$ \\
\hline Azuay & 448 & 83,1 & 15 & 3,3 & 72 & 11,9 & 0 & 0 \\
\hline Bolívar & 81 & 48,0 & 7 & 8,6 & 33 & 18,3 & 1 & 3,0 \\
\hline Carchi & 38 & 25,6 & 1 & 2,6 & 11 & 6,8 & 2 & 18,2 \\
\hline Cañar & 706 & 258,8 & 15 & 2,1 & 21 & 9,9 & 9 & 42,9 \\
\hline Chimborazo & 3140 & 819,8 & 107 & 3,4 & 52 & 12,5 & 1 & 1,9 \\
\hline Cotopaxi & 2177 & 747,9 & 92 & 4,2 & 21 & 7,0 & 1 & 4,8 \\
\hline Imbabura & 4745 & 1633,5 & 85 & 1,8 & 89 & 27,7 & 0 & 0 \\
\hline Loja & 321 & 81,0 & 14 & 4,4 & 107 & 25,4 & 7 & 6,5 \\
\hline Pichincha & 2127 & 109,0 & 34 & 1,6 & 52 & 2,2 & 0 & 0 \\
\hline Tungurahua & 1732 & 444,4 & 47 & 2,7 & 8 & 1,8 & 0 & 0 \\
\hline Total Sierra & 15515 & 326,3 & 417 & 2,7 & 466 & 8,6 & 21 & 4,5 \\
\hline El Oro & 4673 & 1031,8 & 23 & 0,5 & 353 & 65,8 & 0 & 0 \\
\hline Esmeraldas & 5425 & 1911,0 & 52 & 1,0 & 202 & 50,7 & 0 & 0 \\
\hline Guayas & 14951 & 541,2 & 119 & 0,8 & 1252 & 38,2 & 6 & 0,5 \\
\hline Los Ríos & 3814 & 675,8 & 32 & 0,8 & 37 & 5,8 & 0 & 0 \\
\hline Manabí & 1845 & 168,4 & 49 & 2,7 & 1437 & 116,8 & 10 & 0,7 \\
\hline Total Costa & 30708 & 689,1 & 275 & 0,9 & 3281 & 54,0 & 16 & 0,5 \\
\hline Morona & 3 & 2,8 & 1 & 33,3 & 0 & 0 & 0 & 0 \\
\hline Napo & 1 & 0,8 & 0 & 0 & 0 & 0 & 0 & 0 \\
\hline Pastaza & 34 & 71,3 & 1 & 2,9 & 7 & 11,9 & 0 & 0 \\
\hline Sucumbíos & 51 & 53,3 & 1 & 2,0 & 1 & 0,8 & 0 & 0 \\
\hline Zamora & 5 & 6,6 & 2 & 40,0 & 0 & 0 & 0 & 0 \\
\hline Total Oriente & 94 & 21,0 & 5 & 5,3 & 8 & 1,4 & 0 & 0 \\
\hline Islas Galápagos & 3 & 27,8 & 0 & 0 & 0 & 0 & 0 & 0 \\
\hline Total país & 46320 & 441,1 & 697 & 1,5 & 3755 & 30,8 & 37 & 1,0 \\
\hline
\end{tabular}

Fuente: Dirección Nacional de Epidemiología, Ministerio de Salud Pública del Ecuador.

a Tasa anual por 100000 habitantes. 
de V.algynoliticus, 7 de Aeromonas spp., 1 de Campylobacter spp. y 1 de Salmonella enteritidis. En 267 muestras no se aislaron bacterias enteropatógenas y 55 muestras obtenidas con hisopo fueron rechazadas por no cumplir los requisitos establecidos para su procesamiento. Las muestras negativas para $V$. cholerae o positivas para otros enteropatógenos fueron restadas del total de casos de cólera y notificadas como intoxicaciones alimentarias. La confirmación bacteriológica en el laboratorio de referencia PROCED ALA 93/25 demostró que todas las cepas de $V$. cholerae correspondían al serotipo O:1, El Tor, característico de la séptima pandemia, y al subtipo Ogawa. Estos 301 casos confirmados de $V$. cholerae representan $8 \%$ del número total de casos notificados en el país en 1998.

En el cuadro 2 se muestran los resultados de las pruebas de sensibilidad a los antibióticos usados habitualmente en la región. El 100\% de las 301 cepas fueron sensibles a la tetraciclina y quinolonas, y 5,6\% fueron resistentes a la eritromicina. La única cepa resistente a la amoxicilina expresó en realidad un fenotipo multirresistente (a la amoxicilina, amoxicilina/ácido clavulánico, trimetoprim/sulfametoxazol, gentamicina, eritromicina y compuesto vibriostático O/129). Esta cepa se aisló en Quito en un viajero que procedía de Lima por vía terrestre. No hubo discordancia de resultados entre las técnicas utilizadas hasta 1997 y las utilizadas en 1998.

\section{Acciones preventivas}

Con el fin de prevenir la extensión del cólera en el país y reducir la morbilidad y mortalidad por la enfermedad, el MSP, con el apoyo de gobiernos locales y organismos internacionales, entre ellos la OPS/OMS y PROCED ALA 93/25, realizó varias actividades: promoción de la participación comunitaria a través de líderes y voluntarios de salud capacitados para producir hipoclorito de sodio in situ, distribuirlo y usarlo correctamente en la desinfección del agua en el ámbito doméstico; educación familiar y monitoreo de la calidad del agua de consumo humano; implementación de 24 centros de cloración en las áreas de salud de las cinco provincias de la Costa, las más vulnerables, e intensas campañas de educación sanitaria en la prensa escrita y audiovisual.

En colaboración con el Ministerio de Educación, el MSP fortaleció los programas "Escuelas Saludables", promoviendo cambios de comportamiento higiénico-sanitario en la población estudiantil y sus familias, e implementando suministros de agua segura en 294 escuelas con infraestructura deficiente, de los que se beneficiaron aproximadamente 32940 alumnos. También se realizaron campañas de educación entre los expendedores de alimentos sobre la manipulación adecuada de los mismos, se intensificaron las actividades de control sanitario en restaurantes y sitios de venta ambulante de alimentos en las zonas de mayor afluencia turística, y se reforzaron las áreas de salud con unidades móviles para la atención de casos y la promoción de la salud durante los períodos vacacionales en las zonas de mayor movimiento poblacional.

Paralelamente, se fortaleció el sistema de vigilancia epidemiológica con talleres descentralizados de capacitación sobre el nuevo sistema de alerta y epidemiología de intervención, dirigidos a 240 epidemiólogos y trabajadores de la salud; en las unidades operativas se realizaron talleres de actualización del personal de salud para la detección oportuna de casos, el tratamiento adecuado por rehidratación oral o parenteral y la referencia y contrarreferencia de casos, y se mejoró la capacidad resolutiva de la red nacional de laboratorios mediante la formación de 184 técnicos de laboratorio en el diagnóstico bacteriológico precoz de $V$. cholerae, la entrega de insumos (medios de cultivo TCBS y gelosa alcalina y medio de transporte de Cary-Blair) y la elaboración y difusión de manuales de procedimientos $(13,18,19)$.

\section{DISCUSIÓN}

Comparando el brote de cólera de 1998 con la epidemia de 1991, la evolución de la curva de los nuevos casos es de tipo epidémico en ambas ocasiones (figura 1). Existen muchas similitudes témporo-espaciales entre los dos episodios, pero el brote de 1998 tuvo un impacto diez veces menor y el área geográfica afectada fue menos extensa: solo se vieron afectadas 17 de las 21 provincias del país (cuadro 1). Sin embargo, las cuatro provincias que no notificaron casos en 1998 (tres del Oriente y Galápagos) prácticamente tampoco habían sido afectadas por el brote de 1991. La distancia con respecto al epicentro de la enfermedad y la gran dispersión de la población en estas áreas,
CUADRO 2. Sensibilidad a los antibióticos de 301 cepas de Vibrio cholerae aisladas en Ecuador, 1998

\begin{tabular}{|c|c|c|c|c|c|c|}
\hline \multirow[b]{3}{*}{ Antibiótico } & \multicolumn{6}{|c|}{ Sensibilidad } \\
\hline & \multicolumn{2}{|c|}{ Completa } & \multicolumn{2}{|c|}{ Parcial } & \multicolumn{2}{|c|}{ Ninguna } \\
\hline & No. & $\%$ & No. & $\%$ & No. & $\%$ \\
\hline Amoxicilina & 53 & 17,6 & 247 & 82,1 & 1 & 0,3 \\
\hline Tetraciclina & 301 & 100 & 0 & 0 & 0 & 0 \\
\hline Eritromicina & 40 & 13,3 & 244 & 81,1 & 17 & 5,6 \\
\hline Gentamicina & 300 & 99,7 & 0 & 0 & 1 & 0,3 \\
\hline Ácido nalidíxico & 301 & 100 & 0 & 0 & 0 & 0 \\
\hline Norfloxacino & 301 & 100 & 0 & 0 & 0 & 0 \\
\hline Ciprofloxacino & 301 & 100 & 0 & 0 & 0 & 0 \\
\hline Cloranfenicol & 301 & 100 & 0 & 0 & 0 & 0 \\
\hline TMP/SMX & 300 & 99,7 & 0 & 0 & 1 & 0,3 \\
\hline Colistina & 0 & 0 & 0 & 0 & 301 & 100 \\
\hline O/129 & 300 & 99,7 & 0 & 0 & 1 & 0,3 \\
\hline
\end{tabular}

TMP/SMX: trimetoprim/sulfametoxazol, O/129: compuesto vibriostático. 
explicarían la baja incidencia observada en ambas ocasiones (9). Los casos notificados en 1991 se relacionaron con la importación de la enfermedad por trabajadores migrantes (9). La disminución de la incidencia entre 1991 y 1998 y la reducción de la extensión de la enfermedad de la Costa a la Sierra podrían deberse a la inmunocompetencia de la población frente a una enfermedad endémica en el país (9). En cambio, en 1991 la epidemia afectó a una población inmunológicamente virgen después de la ausencia de la enfermedad en el continente durante más de un siglo (1-3). La reducción del impacto y de la difusión del brote también se podría explicar por las medidas de prevención excepcionales llevadas a cabo por las autoridades nacionales y los organismos internacionales. En 1991, la autoridades sanitarias no estaban preparadas para enfrentar la epidemia, pero en 1998 se pusieron en marcha acciones estratégicas tales como la intensificación de las campañas de comunicación, información y educación sanitaria, la provisión de sistemas de agua segura con participación de la comunidad, el fortalecimiento del sistema nacional de vigilancia epidemiológica, la construcción de medios nacionales para el diagnóstico precoz, tanto clínico como de laboratorio, y el tratamiento oportuno de los casos $(13,18,19)$, medidas que permitieron controlar la expansión de la enfermedad y reducir la letalidad global del 1,5\% registrado en 1991 a una cifra ligeramente inferior al 1\%, comparable con la de Perú $(8,13)$. En lo que se refiere a las muestras de pacientes sospechosos de cólera en las que no se aislaron bacterias enteropatógenas, se podría especular que la mayoría de estos casos ya habrían recibido antibióticos antes de la toma de la muestra o que la calidad de la toma y transporte de la misma era inapropiada.

Al igual que en 1991, en 1998 la letalidad fue más elevada en la Sierra que en la Costa (cuadro 1), a pesar de que la incidencia fue menor. En 1991, la mayor letalidad se observó en Zamora y Morona (cuadro 1), provincias del Oriente más alejadas del epicentro de la epidemia (9) y con baja incidencia (17).
En 1998 se repitió la misma tendencia en la Sierra, en las provincias más aisladas de Cañar y Carchi, donde la letalitad alcanzó el 43\% y el 18\%, respectivamente. Esta alta letalidad en áreas que prácticamente no fueron afectadas por el brote de cólera ni por el fenómeno de "El Niño" podría deberse a que estas provincias se caracterizan por una importante dispersión rural, dificultades de acceso a los servicios de salud y un alto porcentaje de población indígena con diferente cultura y grado de alfabetización $(8,12)$, y destaca la necesidad de realizar campañas de educación con cobertura masiva.

En 1998, los primeros casos de cólera se notificaron en la provincia de Loja (12), fronteriza con Perú, pero no se pudo comprobar la procedencia exacta de las cepas, de origen peruano o autóctono, como en febrero del 1991, cuando la enfermedad fue introducida en Ecuador por los trabajadores estacionales de regreso de Perú a la provincia de El Oro $(3,9)$.En 1998, la expansión de la enfermedad estuvo relacionada nuevamente con los intensos movimientos poblacionales característicos del período vacacional de Semana Santa entre las provincias de la Costa de sur a norte y entre la Costa y la Sierra, cuando los trabajadores estacionales de las camaroneras regresan a sus hogares, llevando consigo bebidas y alimentos en condiciones higiénicas inadecuadas (3, 9). La migración de trabajadores estacionales y el consumo de aguas y alimentos potencialmente contaminados son elementos históricamente reconocidos como factores agravantes de la transmisión del cólera en América Latina (8). Sin embargo, estos movimientos poblacionales se observan cada año y no son suficientes para explicar el incremento significativo del número de casos de cólera con respecto al año anterior, cuando solo se registraron 65 casos (8). El fenómeno de "El Niño", por su amplitud, favoreció la reaparición de la enfermedad, no solo en Ecuador y Perú, sino en todo el mundo (8, 10). En Ecuador, la subida de las aguas en los estuarios de las tres provincias costeñas más afectadas, Manabí, Guayas y El Oro (13), pudo explicar la importancia de este nuevo brote. $V$. chole- rae puede multiplicarse a temperaturas de 20 a $40{ }^{\circ} \mathrm{C}$ en condiciones óptimas de salinidad del 1 a 3\%. Su presencia como comensal en el zooplancton, las plantas acuáticas y sus respectivos predadores $(20,21)$, así como las condiciones ecológicas producidas por las inundaciones causadas por "El Niño", podrían haber facilitado el crecimiento de la bacteria en aguas de temperatura adecuada y baja salinidad. A los cambios ecológicos inducidos por "El Niño" se suman la insalubridad ambiental posterior a la destrucción de los sistemas de agua potable, el desbordamiento de alcantarillas o pozos sépticos y los intensos movimientos poblacionales arriba mencionados (13).

Las pruebas de serotipificación de las cepas de $V$. cholerae efectuadas en el laboratorio de referencia muestran que la totalidad de las mismas corresponden a $V$. cholerae O:1, El Tor, característico de la séptima pandemia (4), subtipo Ogawa. En 1991, un 99\% de las cepas correspondían al subtipo Inaba (22). A partir de marzo de 1992 aparecieron las primeras cepas Ogawa $\mathrm{y}$, progresivamente, el subtipo Inaba cedió paso al Ogawa en el país (23). La diferencia entre subtipos depende de la expresión cuantitativa de los antígenos somáticos (24). La interconversión de Ogawa a Inaba es frecuente, y la de Inaba a Ogawa es posible gracias a la presión selectiva producida por la respuesta inmunitaria de los pacientes durante la infección (24).

La vigilancia sistemática de la sensibilidad de $V$. cholerae O:1 debe su importancia al uso racional de los antibióticos como complemento de la rehidratación oral o parenteral en el tratamiento de los casos graves, reduciendo tanto la duración de la enfermedad como la difusión del vibrión (15). Al igual que en 1991, cuando la totalidad de las cepas eran sensibles a los antibióticos usualmente activos (9), y al igual que en un estudio de 122 cepas efectuado entre 1994 y 1996 (15), en 1998 la totalidad de las cepas fueron sensibles a la tetraciclina, a la asociación trimetoprim/sulfametoxazol, a las quinolonas y a los aminoglucósidos. A excepción de la única cepa multirresistente aislada en 1998, la resistencia a la 
amoxicilina fue nula. Sin embargo, se observó un ligero aumento de la resistencia a la eritromicina, de 3\% en 1996 (15) a 5,6\% en 1998 (5,3\% si no se considera la única cepa multirresistente). Globalmente, la sensibilidad de $V$. cholerae 0:1 en Ecuador no ha variado en estos últimos años y el grado de resistencia a los antibióticos utilizados habitualmente queda muy bajo, confirmando la eficacia del tratamiento empírico recomendado por la OMS (25). En 1992 ya se había señalado la existencia de dos cepas multirresistentes en Guayaquil (26). Sin embargo, no se aislaron nuevas cepas multirresistentes hasta 1998. Este fenotipo también se ha observado esporádicamente en Argentina (27), Chile (28) y Honduras (29). Finalmente, la alta sensibilidad de las cepas aisladas en Ecuador en 1998 es comparable con una serie de estudios efectuados en Argentina (30) y Colom- bia (31). Aunque no se justifica cambiar los esquemas de tratamiento vigentes en la Región, se recomienda seguir vigilando la resistencia de $V$. cholerae, aprovechando la incorporación de Ecuador al programa regional de la OPS de vigilancia de la resistencia bacteriana a los antimicrobianos.

La sensibilización y educación de la comunidad, la detección oportuna y el tratamiento adecuado de los casos, la rehidratación sistemática de los pacientes, el apoyo nacional e internacional para mejorar la vigilancia y fortalecer la capacidad resolutiva de los laboratorios, así como la desinfección del agua, contribuyeron a reducir el impacto de la nueva epidemia de cólera en Ecuador, tanto en términos de letalidad como de incidencia.

La información obtenida mediante el estudio de este brote es de suma importancia, aunque podría incremen- tarse su valor utilizando métodos moleculares de caracterización epidemiológica.

Tomando en cuenta la frecuencia de los desastres naturales en Ecuador y su relación con la reaparición del cólera en las zonas fronterizas y la Costa, las deficiencias del abastecimiento de agua, de su calidad y de la infraestructura higiénico-sanitaria, es recomendable que las autoridades de salud diseñen en los próximos años intervenciones que permitan prevenir y controlar la reaparición del cólera y su extensión hacia las provincias más vulnerables de la Sierra y el Oriente.

Agradecimientos. A los doctores Caroline Chang (OPS/OMS, Ecuador), Gabriel Schmunis (OPS/OMS, Washington, D.C.) y José Ramiro Cruz (OPS/ OMS, Washington, D.C.), por sus valiosos aportes.

\section{REFERENCIAS}

1. Boutin JP, Merlin M, Guélain J. L'épidémie de choléra au Pérou en 1991. Bulletin Epidémiologique Hebdomadaire. 1991;49:214-215.

2. Pollitzer R. Cholera. Geneva: World Health Organization; 1959. Pp. 820-892. (Monograph Series 43).

3. Berche P, Weil O. L'épidémie de choléra en Amérique Latine. Med Mal Infect 1993;23: 85-98.

4. Tauxe RV, Blake PA. Epidemic cholera in Latin America. JAMA 1992;267:1388-1390.

5. Centers for Disease Control and Prevention. Cholera - Peru, 1991. MMWR 1991;40:108-110.

6. Centers for Disease Control and Prevention. Update: cholera outbreak - Peru, Ecuador, and Colombia. MMWR 1991;40:225-227.

7. Centers for Disease Control and Prevention. Update: cholera - Western Hemisphere. MMWR 1991;40:860.

8. Organización Panamericana de la Salud. Salud en las Américas (vol. I). Washington, D.C.: OPS; 1998. Pp. 131-136.

9. Weil O, Berche P. The cholera epidemic in Ecuador: towards an endemic in Latin America. Rev Epidemiol Sante Publique 1992;40: 145-155.

10. World Health Organization. Cholera, 1998. Wkly Epidemiol Rec 1999;74:257-263.

11. Delavaud AC. Guayaquil au temps du choléra. Bulletin de l'Institut Français d'Études Andines. 1996;25 (3):509-527.
12. Ecuador, Ministerio de Salud Pública. Informe estadístico epidemiológico. Número de casos y tasas de incidencia anual de cólera según provincias y regiones. Ecuador 19911999. Quito: Dirección Nacional de Epidemiología, MSP; 2000.

13. Ecuador, Ministerio de Salud Pública. Fenómeno de El Niño. Memoria Ecuador 97-98. Quito: MSP; 1999. Pp. 62-67.

14. Dodin A, Fournier JM. Méthodes de laboratoire pour le diagnostic du Vibrion cholérique et des autres Vibrions. Paris: Ed. Institut Pasteur; 1992.

15. Comité de l'Antibiogramme de la Société Française de Microbiologie. Communiqué 1994. Pathol Biol 1994;42, I-VIII.

16. National Committee for Clinical Laboratory Standards. Performance standards for antimicrobial disk susceptibility tests (6th ed.). NCCLS: Wayne, PA; 1997. (Approved Standard NCCLS Document M2-A6).

17. Aldighieri S, Vela E, Pesantes C. Situation de la sensibilité aux antibiotiques de Vibrio cholerae O:1 en Equateur. Med Trop 1997;57:98.

18. Gasparri E, Tassara C, Velasco M (eds.). El Fenómeno de El Niño en el Ecuador, 1997-1999: Del desastre a la prevención. Quito: CISP, SIISE, ECHO; 1999.

19. Chang C, Cañizares R. Proyecto desinfección de agua a nivel domiciliario en las regiones Costa e Insular del Ecuador frente al fenó- meno de El Niño. Informe Técnico. Ecuador 1998-1999. Guayaquil, Ecuador: Organización Panamericana de la Salud; 1999.

20. Véron M. Vibrio cholerae. En: Le Minor L, Véron $\mathrm{M}$, eds. Bactériologie médicale. Paris: Flammarion Médecine-Sciences; 1990. Pp. 476506.

21. Chakraborty S, Nair GB, Shinoda S. Pathogenic vibrios in the natural aquatic environment. Rev Environ Health 1997;12:63-80.

22. Vela E, Chiriboga M, Rivera M, Vásquez R, Burbano L. Aislamiento e identificación de $\mathrm{Vi}$ brio cholerae en la zona Norte del Ecuador. Revista Ecuatoriana de Higiene y Medicina Tropical 1992;40:67-75.

23. Vela E, Escalante L, Escalante S, Rivera M. Vibrio cholerae Inaba cede el paso al Vibrio cholerae Ogawa en la Zona Norte del Ecuador. Boletín Especial de Microbiología. Quito: Ministerio de Salud Pública; 1994;1:41-49.

24. Kaper JB, Morris JG Jr, Levine MM. Cholera. Clin Microbiol Rev 1995;8:48-86.

25. World Health Organization. Guidelines for cholera control. Geneva: WHO; 1993.

26. Threlfall EJ, Said B, Rowe B. Emergence of multiple drug resistance in Vibrio cholerae O:1 El Tor from Ecuador. Lancet 1993;342:1173.

27. Rossi A, Galas M, Binztein N, Rivas M, Caffer MI, Corso A, et al. Unusual multiresistant Vibrio cholerae O:1 El Tor in Argentina. Lancet 1993;342:1172-1173. 
28. Castillo L, Ulloa MT, Martinez MA, Silva W, Seoane M, Maldonado A, et al. Characterization of a multiresistant strain of Vibrio cholerae $\mathrm{O}: 1$, isolated from a case of cholera in Chile. Rev Med Chil 1994;122:986-992.

29. Dubon JM, Palmer CJ, Ager AL, Shor-Posner G, Baum MK. Emergence of multiple drugresistant Vibrio cholerae O:1 in San Pedro Sula, Honduras. Lancet 1997;349:924.
30. Yadon Z, Schmunis G. Sensibilidad de Salmonella, Shigella y Vibrio cholerae a los antimicrobianos en las Américas, 1940-1997. En: Resistencia antimicrobiana en las Américas: magnitud del problema y su contención. Washington, D.C.: Organización Panamericana de la Salud; 2000. Pp. 24-38. (OPS/HCP/HCT/ 163/2000).
31. Robledo CR, Robledo JR. Panorama de la resistencia a los antibióticos en Colombia. Rev Panam Infectol 1999;(supl. 1):S26-S32.

Manuscrito recibido el 26 de marzo de 2002. Aceptado para publicación, tras revisión, el 15 de julio de 2002.

ABSTRACT Objective. To describe the outbreak of cholera that occurred in Ecuador in 1998 during the El Niño weather phenomenon, to present data on the resistance of the circulating strains of Vibrio cholerae to antimicrobial drugs, and to describe the preventive measures taken by health authorities in order to reduce the impact of the disease.

Methods. The epidemiological data came from three sources: 1) the registry of the National Bureau of Epidemiology of the Ministry of Public Health of Ecuador, 2) the registry of the National Institute of Hygiene and Tropical Medicine, and 3) the final report of the Training Program for the Fight against Cholera and Diarrheal Diseases. Isolation, identification, and serotyping was done of $V$. cholerae in the feces samples from $10 \%$ of the suspected cholera cases that were identified between 1 January and 31 December 1998. The suspected cases were defined by the sudden appearance of watery diarrhea, with or without dehydration, in epidemic areas. The strains that were isolated were submitted to a standard antibiogram by the diffusion method, in which the following antibiotics were tested: amoxicillin, tetracycline, trimethoprimsulfamethoxazole, vibriostatic compound $\mathrm{O} / 129$, nalidixic acid, erythromycin, norfloxacin, ciprofloxacin, gentamycin, chloramphenicol, and colistin.

Results. In 1998 there were 3755 cases reported in 17 of the 21 provinces of the country. This corresponds to an incidence rate of 53.96 per 100000 population. Thirtyseven patients died, for a case fatality rate of $0.97 \%$. A total of 301 strains of $V$. cholerae were isolated in the 637 suspected-cholera samples that were processed; all corresponded to $V$. cholerae O1, El Tor, subtype Ogawa. All of the strains were sensitive to tetracycline and to quinolones; $5.6 \%$ of the strains were resistant to erythromycin. The only strain resistant to amoxicillin was multiresistant. Officials in Ecuador implemented a series of preventive measures, and the surveillance system was strengthened in order to reduce the impact of the disease.

Conclusions. The preventive measures helped to reduce the impact of the 1998 cholera epidemic in Ecuador, in terms of both incidence and the case fatality rate. Given the overall sensitivity of the strains to the antimicrobial drugs, there is no reason to change the current treatment regimens in the country. Taking into account the frequency of natural disasters in Ecuador and the relation that they have to the reappearance of cholera, interventions should be designed that make it possible to prevent and control the reappearance of the disease and its spread to the most vulnerable provinces of the central Sierra mountainous region and the eastern part of the country. 